\title{
miR-155 promotes fibroblast-like synoviocyte proliferation and inflammatory cytokine secretion in rheumatoid arthritis by targeting FOXO3a
}

\author{
YAXI WANG $^{1 *}$, TIANYING FENG ${ }^{2 *}$, SHASHA DUAN ${ }^{1}$, YILU SHI $^{1}$, \\ SHULING LI ${ }^{1}$, XIAOSHAN ZHANG ${ }^{1}$ and LEI ZHANG ${ }^{1}$ \\ ${ }^{1}$ Department of Ultrasound, The Affiliated Hospital of Inner Mongolia Medical University, Hohhot, Inner Mongolia 010050; \\ ${ }^{2}$ Department of Medical Ultrasound, Bao'an Central Hospital of Shenzhen, Shenzhen, Guangdong 518102, P.R. China
}

Received December 28, 2018; Accepted September 10, 2019

DOI: $10.3892 / \mathrm{etm} .2019 .8330$

\begin{abstract}
The present study aimed to explore the expression and effects of microRNA (miR)-155 in synovial fibroblasts of patients with rheumatoid arthritis (RA). A total of 89 synovial tissues from RA patients and 49 control synovial tissues were collected, and the levels of miR-155 were measured by reverse transcription quantitative-PCR and western blotting. Fibroblast-like synoviocytes (FLS) were isolated from synovial tissues from the control group and were used to evaluate the roles of miR-155 and forkhead box protein O3a (FOXO3a). MTT assay was used to measure the proliferation of FLS. The expression of miR-155 in RA synovial tissues was significantly higher than that in the control group, but the expression of FOXO3a was significantly lower. In RA synovial tissues, miR-155 expression was negatively correlated with FOXO3a expression, but was positively correlated with the release of inflammatory cytokines interleukin (IL)-1 $\beta$, IL- 6 and tumor necrosis factor- $\alpha$ (TNF- $\alpha$ ). A dual-luciferase reporter system showed that miR-155 inhibited the expression of FOXO3a in FLS cells. miR-155 also promoted secretion of the inflammatory cytokines IL-1 $\beta$, IL- 6 and TNF- $\alpha$ by FLS and proliferation of these cells by targeting FOXO3a.
\end{abstract}

Correspondence to: Dr Xiaoshan Zhang or Dr Lei Zhang, Department of Ultrasound, The Affiliated Hospital of Inner Mongolia Medical University, 1 Tongdao North Road, Hohhot, Inner Mongolia 010050, P.R. China

E-mail: zhangxsh133@163.com

E-mail:974897155@qq.com

*Contributed equally

Key words: microRNA-155, forkhead box protein O3a, rheumatoid arthritis, inflammation

\section{Introduction}

Rheumatoid arthritis (RA) is characterized by chronic arthromeningitis, persistent systemic inflammation and production of autoantibodies, which include rheumatoid factors and cyclic citrullinated peptides. RA can also lead to complications, such as intraarticular cartilage damage, joint dysfunction and cardiovascular and pulmonary conditions $(1,2)$. The incidence of RA in China is $0.5-1 \%$, with $5-50$ new cases per 100,000 annually (3).

The main pathological features of RA are abnormal proliferation of synovial cells, infiltration of inflammatory cells, formation of rheumatoid vasospasm, erosion of cartilage and bone, and ultimately destruction of joints $(4,5)$. Abnormal proliferation of synovial cells in RA causes the cells to proliferate from the original 1-3 layers to 5-6 layers or more. Fibroblast-like synoviocytes (FLS) are the most important type of synovial cells in the arthritic joint (6). There are two ways in which FLS interact with immune cells in RA patients. One is through direct contact between FLS and immune cells and the other is through FLS exposure to immune cell-secreted growth factors and inflammatory cytokines. Both these actions stimulate FLS to produce large amounts of chemokines, angiogenic and proinflammatory growth factors, and matrix metalloproteinases and cathepsins to degrade extracellular matrices and cartilage (7). It would therefore be beneficial to unravel the molecular mechanism of RA disease development through research into the regulation mechanism of inflammation and proliferation in FLS.

MicroRNA (miR)-155 has been shown to be highly expressed in the plasma of RA patients and is a potential biomarker for the diagnosis of RA $(8,9)$. miR-155, may affect the progression of RA by regulating the inflammatory response of monocytes (10) and T cells (11) in RA patients (12). However, the expression of miR-155 in the synovial tissue of RA patients and its effects on proliferation and the secretion of inflammatory cytokines by FLS are yet unknown. In the present study, the expression of miR-155 was examined in the synovial tissue of RA patients, and the effect of its expression on the secretion of inflammatory cytokines and the proliferation of FLS cells was explored. The results of the present study indicated that 
miR-155 expression was increased in the synovial tissue of RA patients, and directly inhibited the expression of FOXO3a. As miR-155 regulated FLS proliferation and inflammatory cytokine secretion, it may be a useful target for the development of new RA treatments.

\section{Materials and methods}

Patients and tissues. A total of 89 synovial tissue specimens were obtained from RA patients who received total knee replacement between January 2016 and October 2017 in The Affiliated Hospital of Inner Mongolia Medical University (Huhot, China). There were 53 male patients and 36 female patients, aged between 40 and 70 years. Specimens from 49 patients, who underwent amputation, were collected as controls. All patients in the control group had no history of RA. Of these patients 36 were male and 13 female and their ages ranged between 45 and 73 years. Exclusion criteria were other rheumatic diseases, autoimmune diseases, tumor, liver and kidney insufficiency, pregnancy or lactation and mental disorders.

The present study was performed with the approval of The Affiliated Hospital of Inner Mongolia Medical University. All aspects of the study complied with the Declaration of Helsinki (13). In addition, all participants signed informed consent forms.

Isolation and culture of FLS. FLS were isolated, cultured and characterized from surgically removed synovial tissue. The detailed experimental methods can be found in Zhang et al (14) FLS cells were cultured in DMEM (Thermo Fisher Scientific, Inc.) which was supplemented with $10 \%$ FBS (cat. no. 10100-147; Thermo Fisher Scientific, Inc.) at $37^{\circ} \mathrm{C}$ with $5 \% \mathrm{CO}_{2}$.

Reverse transcription-quantitative PCR (RT-qPCR). Trizol ${ }^{\circledR}$ (Invitrogen; Thermo Fisher Scientific, Inc.) was used to extract total RNA from tissues or cells. The extracted RNA was reverse transcribed to cDNA using a PrimeScript ${ }^{\mathrm{TM}}$ RT Master Mix reverse transcription kit (cat. no. RR036B, Takara Biotechnology Co., Ltd.) which was performed according to the manufacturer's protocol. Reaction mixture $(20 \mu \mathrm{l})$ was prepared according to the SYBR Green qPCR Master Mix kit (cat. no. 638320, Takara Biotechnology Co., Ltd.) instructions and amplified using an ABI 7500 fluorescence qPCR instrument (Applied Biosystems; Thermo Fisher Scientific Inc.). Thermocycling conditions were: $95^{\circ} \mathrm{C}$ for $30 \mathrm{sec}$; 40 cycles of $90^{\circ} \mathrm{C}$ for $5 \mathrm{sec}$ and $65^{\circ} \mathrm{C}$ for $30 \mathrm{sec}$. The sequences of the PCR primers were as follows: miR-155 forward, 5'-ACACTCCAG CTGGGTTAATGCTAATCGTGATA-3' and reverse, 5'-TGG TGTCGTGGAGTCG-3'; forkhead box protein O3a (FOXO3a) forward, 5'-CGGACAAACGGCTCACTCT-3' and reverse, 5'-GTCGGAGAT TCGTAGCTGGA-3'; tumor necrosis factor- $\alpha$ forward, 5'-CCTCTCTCTAATCAGCCCTCTG-3' and reverse, 5'-GAGGACCTGGGAGTAGATGAG-3'; interleukin (IL)-6 forward, 5'-ACTCACCTCTTCAGAACGAAT TG-3' and reverse, 5'-CCATCTTTGGAAGGTTCAGGT TG-3'; IL-1 $\beta$ forward, 5'-ATGATGGCTTATTACAGTGGC AA-3' and reverse, 5'-GTCGGAGATTCGTAGCTGGA-3'; U6 forward, 5'-CTCGCTTCGGCAGCACA-3' and reverse,
5'-AACGCTTCACGAATTTGCGT-3'; and GAPDH forward, 5'-CTCGCCTAGAGTGAGCTCC-3' and reverse, 5'-AAC TGCTGCGTTGACGGGTATG-3'. U6 was used as a reference gene for miR-155 and GAPDH was used as a reference for all other genes. The $2^{-\Delta \Delta \mathrm{Cq}}$ method was used to calculate quantitative gene expression (15).

Western blotting. RIPA lysis buffer (cat. no. P0013K; Beyotime Institute of Biotechnology) was used to lyse FLS and synovial tissue, and a BCA Protein Assay kit (cat. no. P0010S; Beyotime Institute of Biotechnology) was used to measure lysate protein concentration. A total of $50 \mu \mathrm{g}$ protein from tissue or cell lysates was separated using SDS-PAGE on a $10 \%$ gel and then transferred to a PVDF membrane. The membrane was blocked with $5 \%$ skim milk powder for $1 \mathrm{~h}$ at room temperature. The primary antibodies used were anti-FOXO3a (cat. no. ab70314; 1:5,000) or anti-GAPDH (cat. no. ab9484; 1:3,000). The secondary antibodies used were horseradish peroxidase-conjugated goat anti-rabbit (cat. no. ab6721; 1:1,000), or goat anti-mouse (cat. no. ab205719; 1:1,000). All antibodies were supplied by Abcam. Membranes were incubated with primary antibodies overnight at $4^{\circ} \mathrm{C}$ and with secondary antibodies for $1 \mathrm{~h}$ at room temperature. The BeyoECL Plus kit (cat. no. P0018S; Beyotime Institute of Biotechnology) was used to visualize protein bands and densitometry was performed using the Beckman Coulter Immunoassay system (UniCel DxI 800; Beckman Coulter, Inc.).

Cell transfection. Small interfering (si)-FOXO3a RNA (forward, 5'-CCCAUGCUATUGUUGUCACUTT-3' and reverse, 5'-AGUGAUTCGAUGAAUGGGTT-3') and si-negative control (NC) (forward, 5'-ACCGCAUAGUGU AACUUUATT-3' and reverse, 5'-GAAAGUUAGACUAUG CGGCTT-3') were designed and synthesized by Shanghai Shenggong Biology Engineering Technology Service, Ltd. miR-155-mimic (5'-UUA AUGCUA AUCGUGAUA GGGU-3'), miR-155-inhibitor (5'-AAUUACGAUUAGCAC UAUCCCA-3') and miR-155-NC (5'-GCAUUUGAGAGC CAUUAUGGUA-3') were also synthesized by Shanghai Shenggong Biology Engineering Technology Service, Ltd. si-RNA (50 nM), si-NC (50 nM), miR-155-mimic (50 nM), miR-155-inhibitor (50 nM) and miR-155-NC (50 nM) were directly transfected into cells using Lipofectamine ${ }^{\mathrm{TM}} 2000$ transfection reagent (cat. no. 11668019; Invitrogen; Thermo Fisher Scientific Inc.). Wild type or mutant 3'-untranslated regions of FOXO3a were first cloned into the plasmid psiCHECK2 (Promega Corporation) and then transfected into cells as si-RNA. pLV-FOXO3a lentivirus (cat. no. sc-425192; Santa Cruz Biotechnology, Inc.) and its supporting no-load lentivirus (pLV-FOXO3a) were added directly to the FLS cell culture medium, and the number of lentiviral particles used for transfection was 2 fold the number of FLS cells. There was a $72 \mathrm{~h}$ interval between transfection and subsequent experimentation.

Dual-luciferase reporter assay. TargetScan (http://www. targetscan.org) was used to predict the target genes of miR-155. The Dual-Lucy Assay kit (cat. no. D00100; Beijing Solarbio Science \& Technology Co., Ltd.) was used to detect the activity of luciferase following the manufacturer's protocol. 
Briefly, cells were collected after $48 \mathrm{~h}$ of transfection and then lysed for $5 \mathrm{~min}$ on ice before centrifugation $(12,000 \mathrm{x} \mathrm{g}$ for $1 \mathrm{~min}$ at room temperature) to collect the cell supernatant. Five volumes of firefly luciferase reaction solution or Renilla luciferase reaction solution were added to the cell lysate and the enzyme activity was detected.

Cytokine detection. After 48-h incubation with transfection reagents, serum-free medium was added for $24 \mathrm{~h}$. The culture media were then collected and centrifuged at 1,200 x $\mathrm{g}$ for $10 \mathrm{~min}$ at room temperature. A human IL-1 $\beta$ ELISA kit (cat. no. ab46631) was used to determine IL-1 $\beta$ concentration in medium, an IL-6 kit (cat. no. ab46027) to determine IL-6 concentration and a TNF- $\alpha$ kit (cat. no. ab181421) to determine TNF- $\alpha$ concentration. All kits were supplied by Abcam and used according to the manufacturer's instructions.

MTT assay. Cells at a density of $2 \times 10^{3}$ cells/well were seeded in a 96-well culture plate containing DMEM supplemented with $10 \%$ FBS. The viability of FLS cells was assessed by MTT assay. After $4 \mathrm{~h}$ of culture, MTT (10 $\mu \mathrm{l}$ per well, $10 \mathrm{mg} / \mathrm{ml})$ was added to the cells and incubated. Cell supernatant was removed and $100 \mu \mathrm{l}$ DMSO was added. After $30 \mathrm{~min}$, optical density was measured using a plate reader (ELx808; Bio-Tek Instruments, Inc.).

Statistical analysis. Data are presented as the mean \pm SD and were analyzed using SPSS 20.0 (IBM Corp.). Student's t-test was used to compare differences between two groups and one-way ANOVA with Tukey-Kramer post-hoc test was used to compare differences between multiple groups. The correlation between two groups was analyzed by Pearson's correlation coefficient. $\mathrm{P}<0.05$ indicated statistical significance.

\section{Results}

Expression of miR-155 and FOXO3a in synovial tissue. RT-qPCR was used to determine the expression of miR-155 and FOXO3a mRNA in 89 RA synovial tissues and 49 normal synovial tissues. As seen in Fig. 1A, the expression of miR-155 in RA synovial tissues was significantly higher than that in the control group. The expression of FOXO3a mRNA in RA synovial tissues was significantly lower than that in the control group (Fig. 1B). The expression of FOXO3a was also measured by western blotting and the results indicated that the expression level of FOXO3a protein in RA synovial tissues was significantly lower than that in the control group (Fig. 1C and F). miR-155 levels were negatively correlated with FOXO3a protein expression (Fig. 1D) in RA synovial tissues.

The results of a dual luciferase reporter system (Fig. 1E and $\mathrm{G}$ ) indicated that FOXO3a was a target gene of miR-155 in FLS cells, and that a miR-155-mimic could inhibit the expression of FOXO3a while a miR-155-inhibitor could promote FOXO3a expression. These results suggested that altered expression of miR-155 and FOXO3a in RA patients' synovial tissue may have a role in the development of RA.

miR-155 and FOXO3a are associated with inflammation in synovial tissue. The relationship between the expression of miR-155 and FOXO3a in synovial tissue of RA patients and the expression of inflammatory cytokines was analyzed (Fig. 2), and the results suggested that the expression of IL-1 $\beta$, IL-6 and TNF- $\alpha$ mRNA in synovial tissue were positively correlated with miR-155 expression and negatively correlated with FOXO3a expression. This suggested that miR-155 may regulate inflammation in RA synovial tissue by targeting FOXO3a.

miR-155 promotes inflammatory cytokine secretion in FLS cells by targeting FOXO3a. As presented in Fig. 3A, miR-155-mimic increased the expression of miR-155, and miR-155-inhibitor decreased the expression of miR-155. As described in Fig. 3B, miR-155-mimic and si-FOXO3a could reduce the expression of $\mathrm{FOXO} 3$ a protein compared with si-NC, and miR-155-inhibitor and pLV-FOXO3a could promote the expression of $\mathrm{FOXO} 3$ a protein compared with $\mathrm{NC}$ and pLV-NC groups, respectively. The results shown in Fig. 3C and D suggested that miR-155-mimic (vs. NC group) and si-FOXO3a (vs. si-NC group) could reduce the secretion of IL-1 $\beta$, IL- 6 and TNF- $\alpha$ by FLS cells, and that miR-155-inhibitor (vs. NC group) and pLV-FOXO3a (vs. si-NC group) could promote the secretion of IL-1 $\beta$, IL- 6 and TNF- $\alpha$ by FLS cells targeting FOXO3a. This indicated that miR-155 may promote inflammatory cytokine secretion in FLS cells by targeting FOXO3a.

miR-155 promotes FLS cells proliferation by targeting FOXO3a. The effect of miR-155 expression on the proliferation of FLS was investigated, and the results indicated that miR-155-mimic (vs. NC group) and si-FOXO3a (vs. si-NC group) could promote FLS proliferation, and miR-155-inhibitor (vs. NC group) and pLV-FOXO3a (vs. si-NC group) could suppress FLS proliferation (Fig. 4). This indicated that miR-155 regulated FLS cell proliferation by targeting FOXO3a.

\section{Discussion}

miRNAs are a class of evolutionarily conserved, endogenous, non-coding single-stranded RNAs of approximately 20-24 nucleotides in length that regulate gene expression at the post-transcriptional level through cleavage of complementary mRNA or by inhibition of mRNA translation $(7,16)$. Previous studies confirmed that these miRNAs not only participate in regulation of inflammatory responses in RA patients, but also regulate proliferation, apoptosis, invasion and secretion of inflammatory cytokines in FLS $(17,18)$. In a previous study, miR-17 suppressed TNF- $\alpha$ signaling by interfering with tumor necrosis factor receptor-associated factor 2 and inhibitor of apoptosis cIAP2 association (19). An additional study suggested that miR-29a inhibited the proliferation of FLS and induced their apoptosis in RA patients (20). Further studies showed that miR-20a negatively regulated the expression of the NACHT LRR an PYD domain containing protein 3 inflammatory corpuscles in FLS of RA patients by targeting thioredoxin interacting protein (21) and that miR-140-5p inhibited synovial fibroblast proliferation and inflammatory cytokine secretion through targeting toll-like receptor 4 (22). This led to the hypothesis that miRNAs play an important role in the development of RA and are a potential target for the treatment of RA patients. 

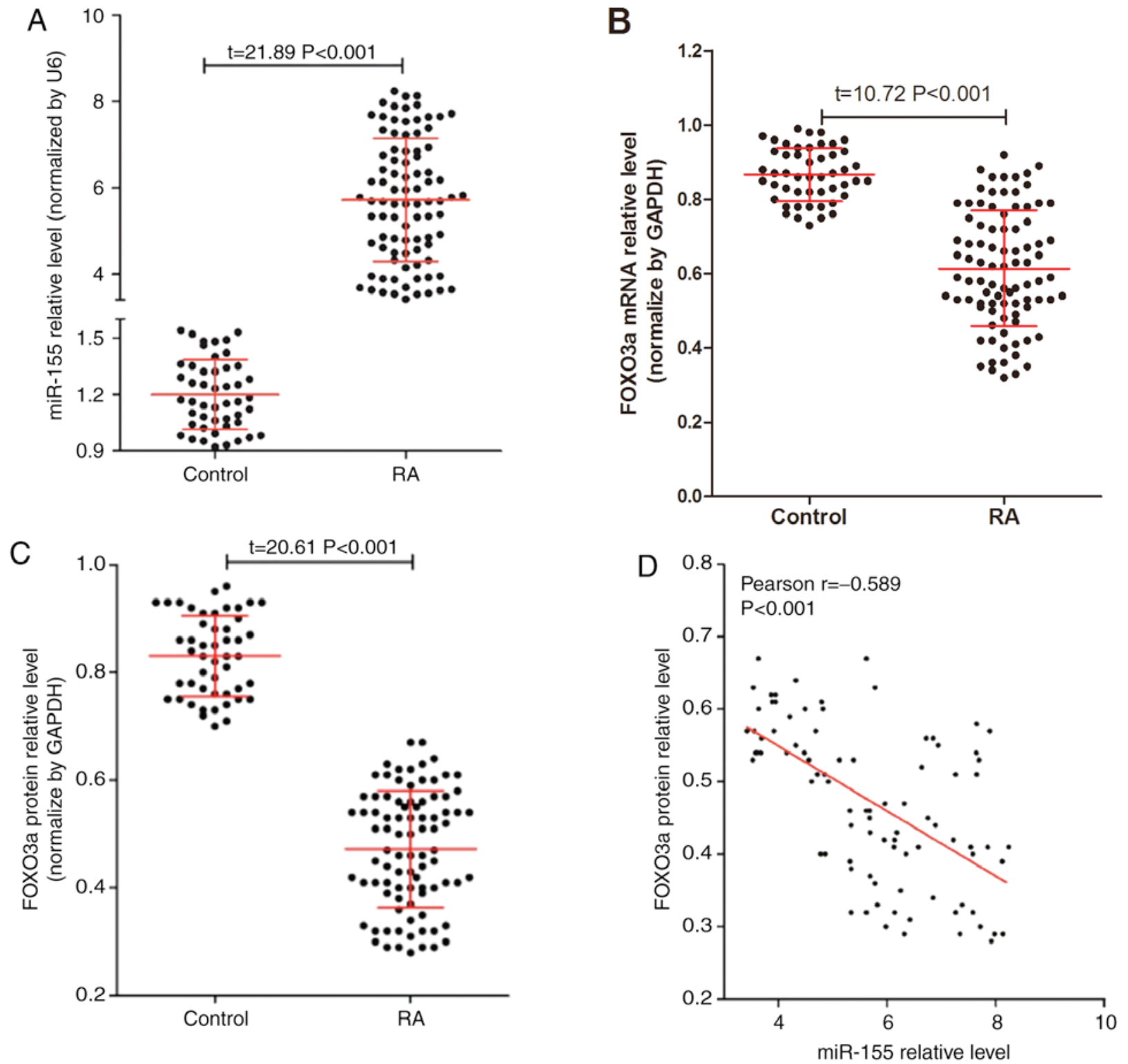

E FОXО3а (WT) 3'-AATTACGA---GTGGAC--ATTC-5'
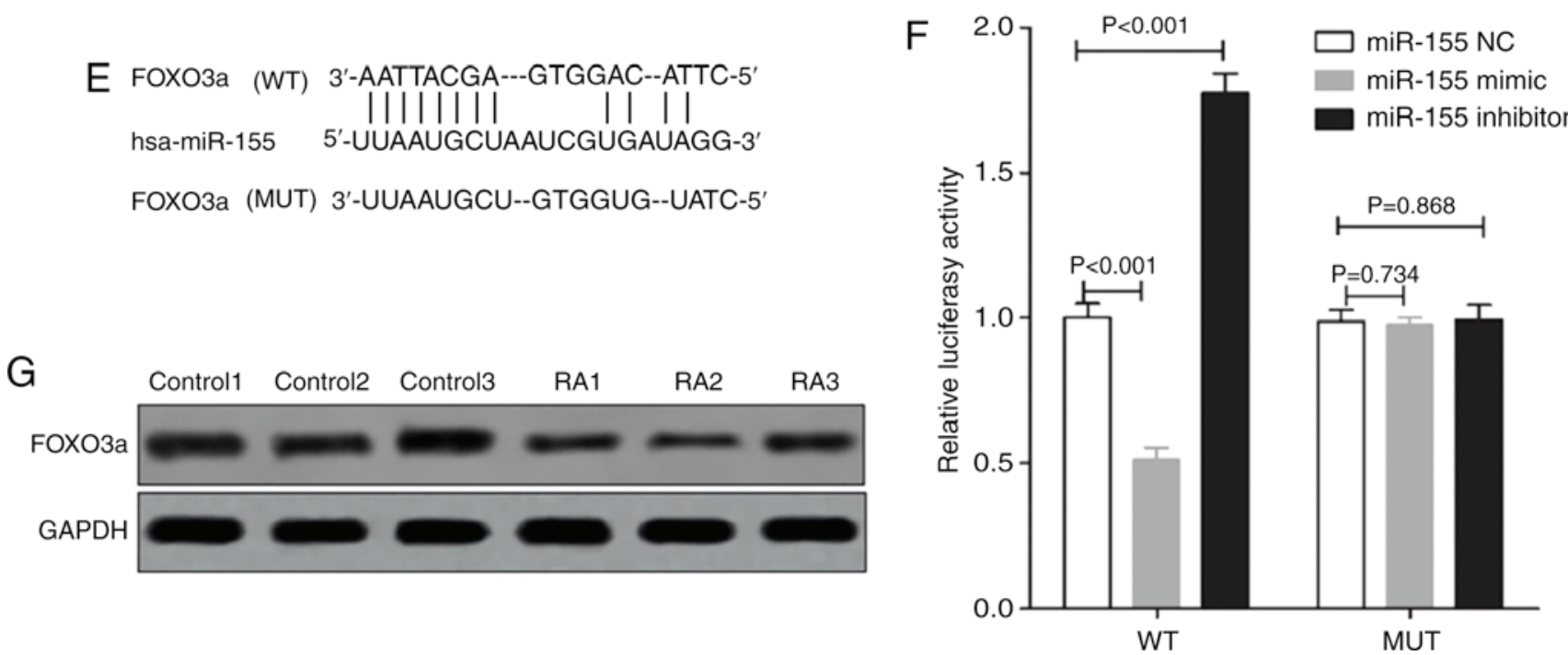

FОXОЗа (MUT) 3'-UUAAUGCU--GTGGUG--UATC-5'

Figure 1. miR-155 and FOXO3a levels in synovial tissue. Reverse transcription-quantitative PCR was used to measure the expression of (A) miR-155 and (B) FOXO3a mRNA in normal and RA synovial tissue. (C) The expression level of FOXO3a protein was quantified in normal and RA synovial tissue. (D) Correlation between miR-155 level and FOXO3a protein in RA synovial tissue. (E) WT-FOXO3a 3'-UTR and MUT-FOXO3a 3'-UTR luciferase reporter vectors were constructed; (F) miR-NC/miR-155-mimic/miR-155-inhibitor were transected into FLS cells, and luciferase activity was detected. (G) Representative western blots used to quantify FOXO3a protein levels. 3'-UTR, 3'-untranslated region; FLS, fibroblast-like synoviocyte; FOXO3a, forkhead box protein O3a; miR, microRNA; MUT, mutant; NC, negative control; WT, wild-type. 
A

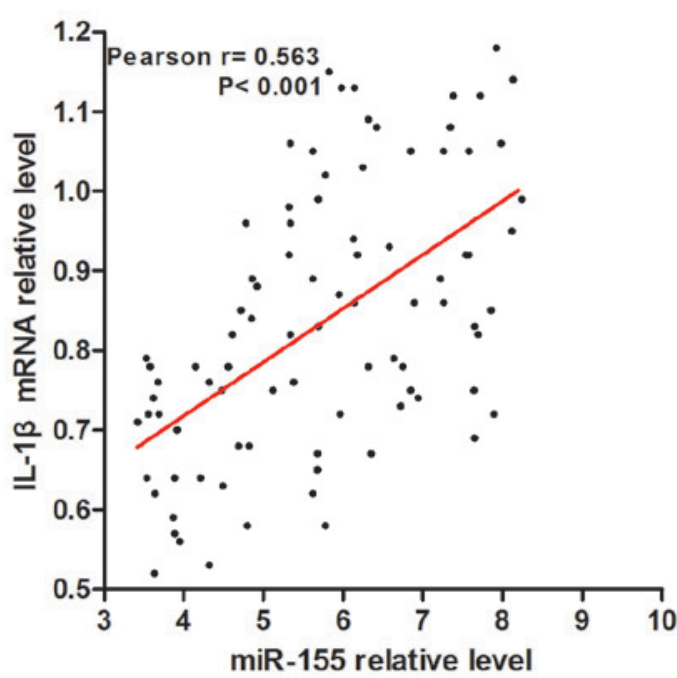

C

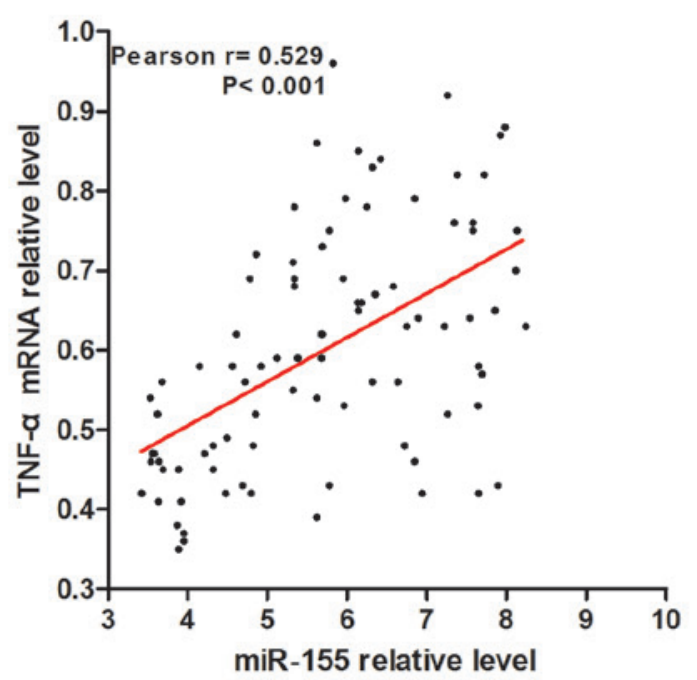

E

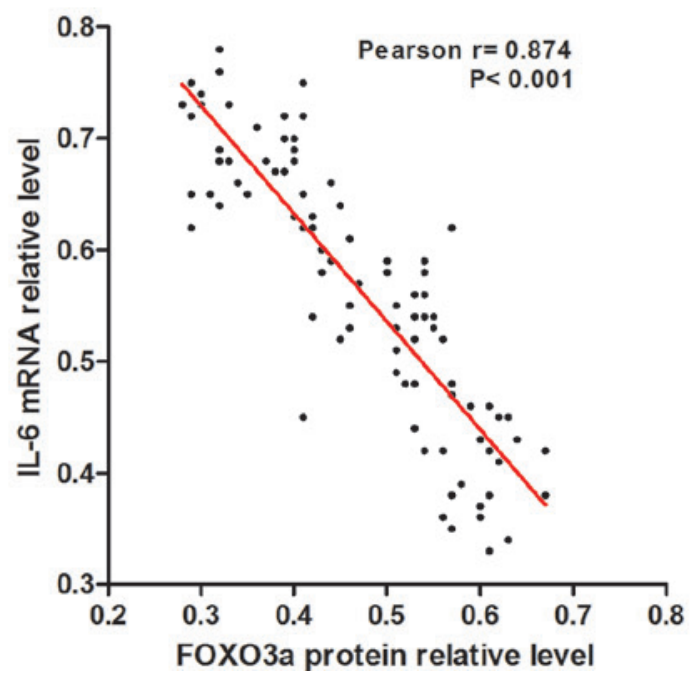

B

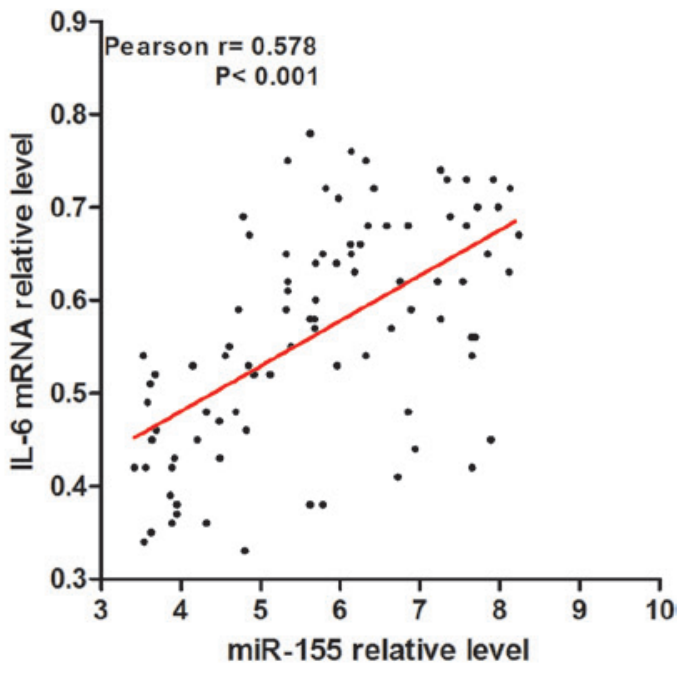

D

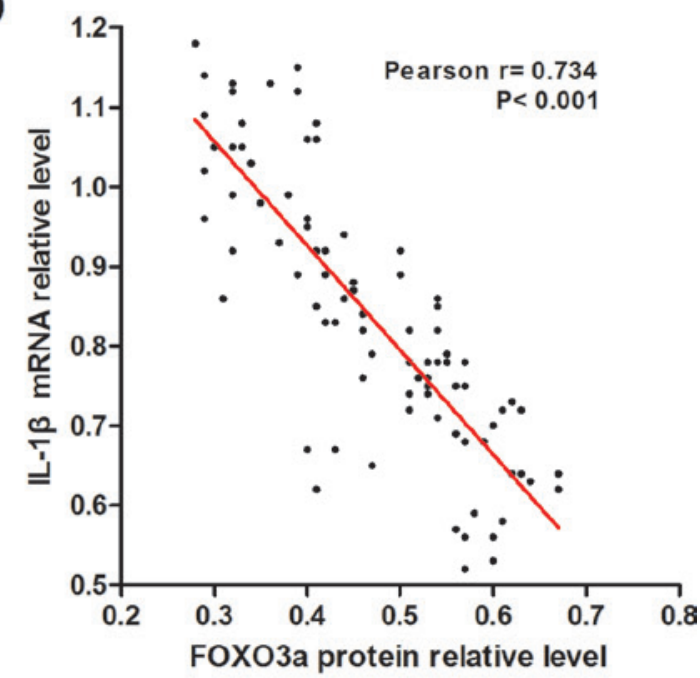

$\mathbf{F}$

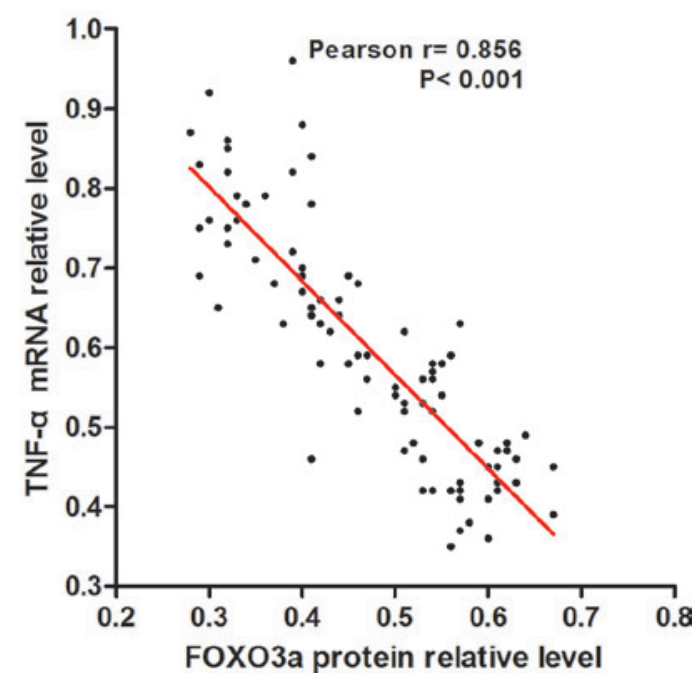

Figure 2. miR-155 and FOXO3a correlation with inflammatory cytokine release. Correlation between the expression of miR-155 and (A) IL-1 $\beta$, (B) IL-6, (C) TNF- $\alpha$ mRNA in synovial tissue. Correlation between the expression of FOXO3a and the release of (D) IL-1 $\beta$, (E) IL-6 and (F) TNF- $\alpha$ from synovial tissue. FOXO3a, forkhead box protein O3A; IL, interleukin; miR, microRNA; TNF- $\alpha$, tumor necrosis factor- $\alpha$. 


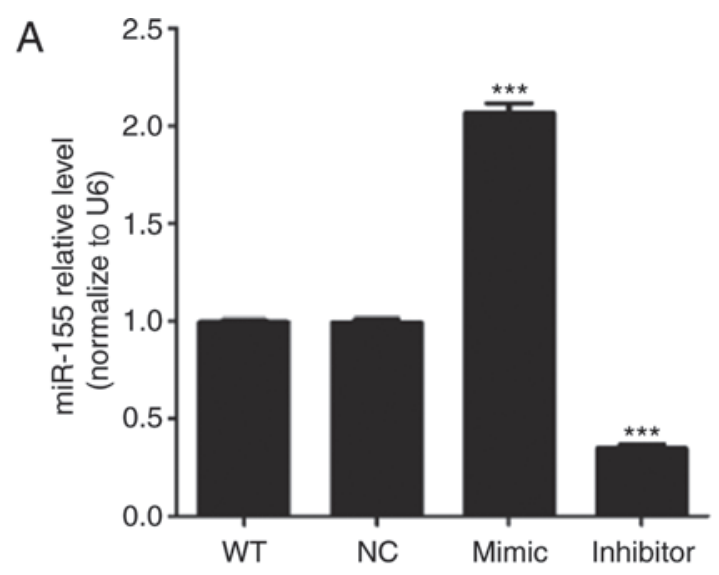

B
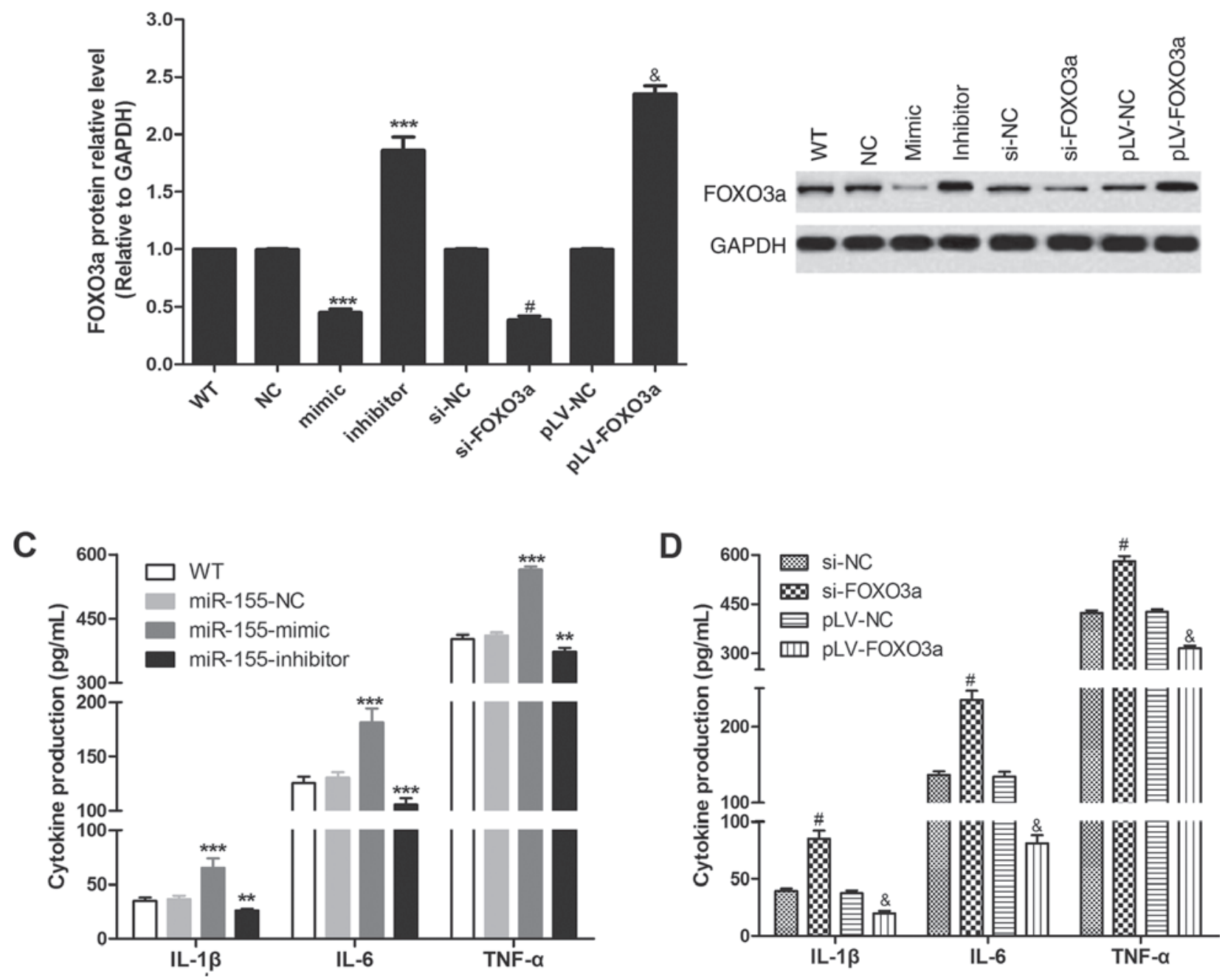

Figure 3. miR-155 and FOXO3a regulate the secretion of inflammatory cytokines by FLS cells. (A) Reverse transcription-quantitative PCR was used to detect the expression of miR-155. (B) Western blot was used to determine the expression of FOXO3a protein in FLS cells after transfection. (C) Cytokine release by FLS in response to miR-mimic and inhibitor. (D) Cytokine release by FLS in response to FOXO3a knockdown and overexpression. All experiments were performed in three independent replicates. ${ }^{* *} \mathrm{P}<0.01$ and ${ }^{* * *} \mathrm{P}<0.001$ vs. miR-155-NC group, ${ }^{\#} \mathrm{P}<0.001$ vs. si-FOXO3a group and ${ }^{\&} \mathrm{P}<0.001$ vs. pLV-NC group. FLS, fibroblast-like synoviocyte; FOXO3a, forkhead box protein O3a; interleukin, IL; LV, lentivirus; miR, microRNA; NC, negative control; si, small interfering; TNF- $\alpha$, tumor necrosis factor- $\alpha$; WT, cells without any treatment.

Large numbers of infiltrating inflammatory cells and overexpression of inflammatory cytokines are some of the main causes of RA disease progression $(23,24)$. In the present study, increased levels of miR-155 were found in synovial tissue of RA patients and were negatively correlated with FOXO3a protein expression. As miR-155 is a non-coding RNA, it cannot directly regulate the biological behavior of cells, and may participate indirectly through regulation of target gene expression (25). FOXO3a is a target gene of miR-155, and miR-155 can regulate glucose metabolism (26), and 

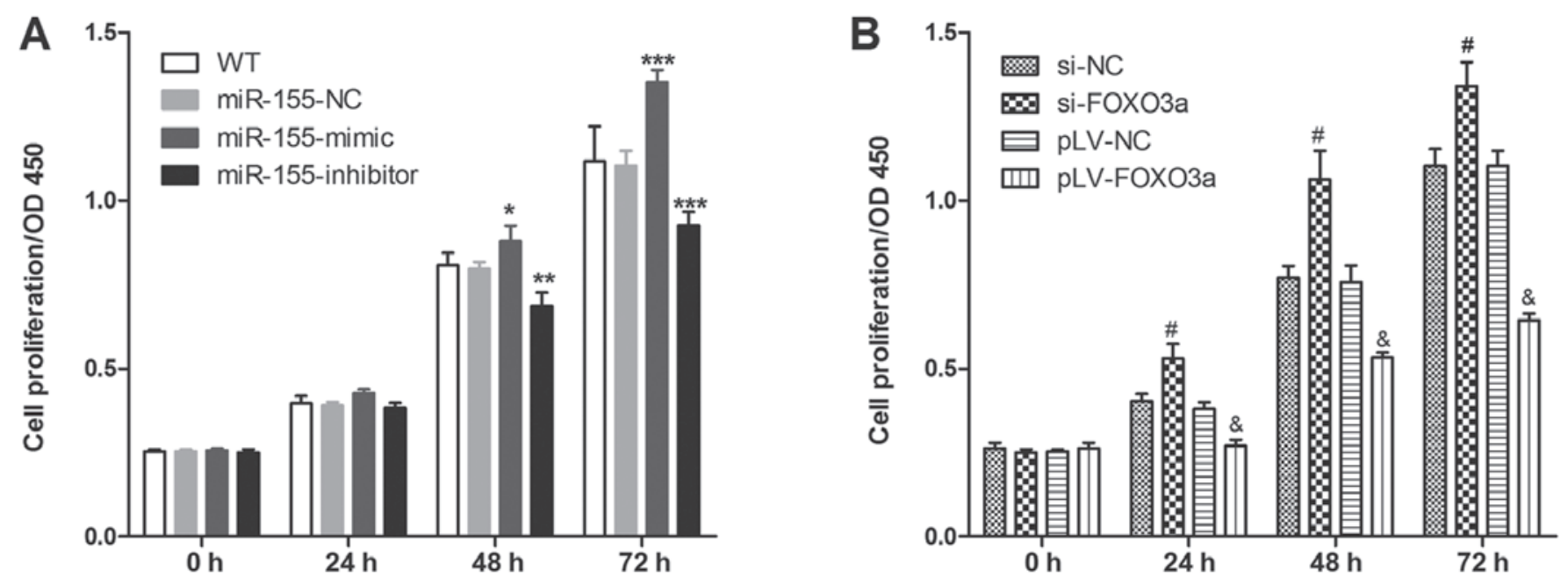

Figure 4. miR-155 and FOXO3a regulate FLS cell proliferation. MTT assay was used to detect FLS cell proliferation after (A) treatment influencing miR-155 expression and (B) treatment influencing FOXO3a expression. Three independent experiments were performed. ${ }^{*} \mathrm{P}<0.05$, ${ }^{* *} \mathrm{P}<0.01$ and ${ }^{* * *} \mathrm{P}<0.001$ vs. miR-155-NC group, ${ }^{\# P<0.001 ~ v s . ~ s i-N C ~ g r o u p ~ g r o u p ~ a n d ~}{ }^{\circledR} \mathrm{P}<0.001$ vs. pLV-NC group. FOXO3a, forkhead box protein O3a; FLS, fibroblast-like synoviocyte; LV, lentivirus; miR, microRNA; NC, negative control; MUT, mutant; si, small interfering RNA; WT, cells without any treatment.

proliferation, migration, invasion $(27,28)$ and apoptosis (29) of cancer cells though inhibition of FOXO3a expression. The miR-155/FOXO3a axis is known to play an important role in the pathogenesis of inflammatory disease (30). A dual luciferase reporter gene system was used in the present study to verify that miR-155 targeted the inhibition of FOXO3a expression in FLS.

RA is a chronic, systemic disease characterized by inflammatory synovitis, and multiple inflammatory factors are involved in the pathological progression of RA, such as IL-1 $\beta$, IL-6 and TNF- $\alpha$ (31,32). Previous studies confirmed that miR-155, which is highly expressed in the circulation of RA patients, could affect the peripheral blood inflammatory response of RA patients by regulating peripheral blood mononuclear cells (10) and T cell phenotypes (11), and that inhibition of miR-155 expression in the peripheral blood of patients with RA would help to improve the condition $(11,12)$. The results of the present study indicate that miR-155 levels are positively correlated with inflammatory cytokine release (IL-1 $\beta$, IL- 6 and TNF- $\alpha$ ) by synovial tissue of RA patients. It has been reported that treatment with an IL-1 $\beta$ antibody may significantly alleviate RA symptoms (33). An additional study indicated that inhibition of IL-1 secretion is a possible method for the treatment of RA (34). Further research suggests that anti-IL-6 receptor-related drugs can be used to treat RA, either alone or in combination with other drugs (35), and that inhibition of the biosynthesis of TNF- $\alpha$ could improve RA (36).

Synovial thickening is one of the typical lesions of RA (37). Activated FLS cells have tumor-like proliferation characteristics, resulting in a large increase in the number of cells and synovial thickening (38). The results of the present study also suggested that miR-155 regulated FLS proliferation and inflammatory cytokine secretion in FLS by targeting FOXO3a in vitro. The FOXO family is an important family of transcriptional regulator proteins involved in many cellular functions. Scientists have identified four family members, FOXO1, FOXO3a, FOXO4 and FOXO6 (39). Although each FOXO family member has its own role, FOXO3a has been extensively studied due to its important regulatory effects on cell proliferation, apoptosis, metabolism, and oxidative stress (39). It has been reported that abnormal expression of FOXO3a is closely related to the progression of various types of cancer (40), fibrosis (41) and other diseases $(41,42)$. FOXO3a plays an important role in both inflammation and regulation of cell proliferation. FOXO3a regulates inflammation by $\mathrm{NF}-\kappa \mathrm{B}, \mathrm{T}$ cells and autoinflammation $(43,44)$; however, down-regulation of FOXO3a expression may promote breast cancer cell proliferation (45) and the AKT/FOXO3a signaling pathway plays an important role in the proliferation of human neural cells (46), primary macrophages (47) and fibroblasts (47). These findings indicate that miR-155 may be a potential target for the development of inhibitors of inflammation in patients with RA.

An important limitation of the present study is that all tissue was taken from RA patients who had received total knee replacement and these findings may not be applicable to all forms of RA.

\section{Acknowledgements}

Not applicable.

\section{Funding}

The present study was supported by the Science Foundation of Inner Mongolia [2016MS (LH) 0808].

\section{Availability of data and materials}

The datasets used and/or analyzed during the current study are available from the corresponding author on reasonable request. 


\section{Authors' contributions}

XSZ conceived and designed the present study, and contributed to writing the manuscript. YW and TYF analyzed experimental data. YW, TYF, SSD, SLL, LZ and YLS performed the experiments. All authors read and approved the final manuscript.

\section{Ethics approval and consent to participate}

The present study was approved by the Ethics Committee of The Affiliated Hospital of Inner Mongolia Medical University. All participants signed informed consent forms.

\section{Patient consent for publication}

Not applicable.

\section{Competing interests}

The authors declare that they have no competing interests.

\section{References}

1. Scott DL, Wolfe F and Huizinga TW: Rheumatoid arthritis. Lancet 376: 1094-1108, 2010.

2. Felson DT, Anderson JJ, Boers M, Bombardier C, Furst D, Goldsmith C, Katz LM, Lightfoot R Jr, Paulus H, Strand V, et al: American college of Rheumatology. Preliminary definition of improvement in rheumatoid arthritis. Arthritis Rheum 38: 727-735, 1995.

3. Zeng XF, Zhu SL, Tan AC and Xie XP: Disease burden and quality of life of rheumatoid arthritis in China: A systematic review. Chin J Evidence-Based Med 13: 300-307, 2013.

4. Feldmann M and Maini SR: Role of cytokines in rheumatoid arthritis: An education in pathophysiology and therapeutics. Immunol Rev 223: 7-19, 2008.

5. Firestein GS: Invasive fibroblast-like synoviocytes in rheumatoid arthritis. Passive responders or transformed aggressors? Arthritis Rheum 39: 1781-1790, 1996.

6. Bartok B and Firestein GS: Fibroblast-like synoviocytes: Key effector cells in rheumatoid arthritis. Immunol Rev 233: 233-255, 2010.

7. Noss EH and Brenner MB: The role and therapeutic implications of fibroblast-like synoviocytes in inflammation and cartilage erosion in rheumatoid arthritis. Immunol Rev 223: 252-270, 2008.

8. Abdul-Maksoud RS, Sediq AM, Kattaia A, Elsayed W, Ezzeldin N, Abdel Galil SM and Ibrahem RA: Serum miR-210 and miR-155 expression levels as novel biomarkers for rheumatoid arthritis diagnosis. Br J Biomed Sci 74: 209-213, 2017.

9. Mookherjee N and El-Gabalawy HS: High degree of correlation between whole blood and PBMC expression levels of miR-155 and miR-146a in healthy controls and rheumatoid arthritis patients. J Immunol Methods 400-401: 106-110, 2013.

10. Rajasekhar M, Olsson AM, Steel KJ, Georgouli M, Ranasinghe U, Brender Read C, Frederiksen KS and Taams LS: MicroRNA-155 contributes to enhanced resistance to apoptosis in monocytes from patients with rheumatoid arthritis. J Autoimmun 79: 53-62, 2017.

11. Spoerl D, Duroux-Richard I, Louis-Plence P and Jorgensen C: The role of miR-155 in regulatory $\mathrm{T}$ cells and rheumatoid arthritis. Clin Immunol 148: 56-65, 2013.

12. Leah E: Rheumatoid arthritis: miR-155 mediates inflammation. Nat Rev Rheumatol 7: 437, 2011.

13. La Vaque TJ and Rossiter T: The ethical use of placebo controls in clinical research: The Declaration of Helsinki. Appl Psychophysiol Biofeedback 26: 23-37, 61-65, 2001.

14. Zhang W, Chen L, Jiang Y and Shen Y: miR-26a-5p regulates synovial fibroblast invasion in patients with rheumatoid arthritis by targeting Smad 1. Med Sci Monit 24: 5178-5184, 2018.

15. Liu Y, Qian K, Li C, Ma Y and Chen X: Roles of microRNA-539 and osteopontin in rheumatoid arthritis. Exp Ther Med 15: 2681-2687, 2018
16. Bartel DP: MicroRNAs: Genomics, biogenesis, mechanism, and function. Cell 116: 281-297, 2004.

17. Tavasolian F, Abdollahi E, Rezaei R, Momtazi-Borojeni AA, Henrotin Y and Sahebkar A: Altered expression of MicroRNAs in rheumatoid arthritis. J Cell Biochem 119: 478-487, 2018.

18. Murata K, Yoshitomi H, Tanida S, Ishikawa M, Nishitani K, Ito $\mathrm{H}$ and Nakamura T: Plasma and synovial fluid microRNAs as potential biomarkers of rheumatoid arthritis and osteoarthritis. Arthritis Res Ther 12: R86, 2010.

19. Akhtar N, Singh AK and Ahmed S: MicroRNA-17 suppresses TNF- $\alpha$ signaling by interfering with TRAF2 and cIAP2 association in rheumatoid arthritis synovial fibroblasts. J Immunol 197: 2219-2228, 2016

20. Liu J, Fei D, Xing J and Du J: MicroRNA-29a inhibits proliferation and induces apoptosis in rheumatoid arthritis fibroblast-like synoviocytes by repressing STAT3. Biomed Pharmacother 96: 173-181, 2017.

21. Li XF, Shen WW, Sun YY, Li WX, Sun ZH, Liu YH, Zhang L, Huang C, Meng XM and Li J: MicroRNA-20a negatively regulates expression of NLRP3-inflammasome by targeting TXNIP in adjuvant-induced arthritis fibroblast-like synoviocytes. Joint Bone Spine 83: 695-700, 2016.

22. Li H, Guan SB, Lu Y and Wang F: MiR-140-5p inhibits synovial fibroblasts proliferation and inflammatory cytokines secretion through targeting TLR4. Biomed Pharmacother 96: 208-214, 2017.

23. Innala L, Sjöberg C, Möller B, Ljung L, Smedby T, Södergren A, Magnusson S, Rantapää-Dahlqvist S and Wallberg-Jonsson S: Co-morbidity in patients with early rheumatoid arthritis-inflammation matters. Arthritis Res Ther 18: 33, 2016.

24. Sharma AR, Sharma G, Lee SS and Chakraborty C: miRNA-regulated key components of cytokine signaling pathways and inflammation in rheumatoid arthritis. Med Res Rev 36: 425-439, 2016.

25. Ritchie W, Rasko JE and Flamant S: MicroRNA target prediction and validation. Adv Exp Med Biol 774: 39-53, 2013.

26. Kim S, Lee E, Jung J, Lee JW, Kim HJ, Kim J, Yoo HJ, Lee HJ, Chae SY, Jeon SM, et al: microRNA-155 positively regulates glucose metabolism via PIK3R1-FOXO3a-cMYC axis in breast cancer. Oncogene 37: 2982-2991, 2018.

27. Kong W, He L, Coppola M, Guo J, Esposito NN, Coppola D and Cheng JQ: MicroRNA-155 regulates cell survival, growth and chemosensitivity by targeting FOXO3a in breast cancer. J Biol Chem 291: 22855, 2016

28. Ling N, Gu J, Lei Z, Li M, Zhao J, Zhang HT and Li X: microRNA-155 regulates cell proliferation and invasion by targeting FOXO3a in glioma. Oncol Rep 30: 2111-2118, 2013.

29. Wang Q, Li C, Zhu Z, Teng Y, Che X, Wang Y, Ma Y, Wang Y, Zheng H, Liu Y and Qu X: miR-155-5p antagonizes the apoptotic effect of bufalin in triple-negative breast cancer cells. Anticancer Drugs 27: 9-16, 2016.

30. Min M, Peng L, Yang Y, Guo M, Wang W and Sun G: MicroRNA-155 is involved in the pathogenesis of ulcerative colitis by targeting FOXO3a. Inflamm Bowel Dis 20: 652-659, 2014.

31. Choy EH and Panayi GS: Cytokine pathways and joint inflammation in rheumatoid arthritis. N Engl J Med 344: 907-916, 2001.

32. Araki Y and Mimura T: The mechanisms underlying chronic inflammation in rheumatoid arthritis from the perspective of the epigenetic landscape. J Immunol Res 2016: 6290682, 2016.

33. Qi J, Ye X, Ren G, Kan F, Zhang Y, Guo M, Zhang Z and Li D: Pharmacological efficacy of anti-IL-1 $\beta$ scFv, Fab and full-length antibodies in treatment of rheumatoid arthritis. Mol Immunol 57: 59-65, 2014.

34. Ruscitti P, Cipriani P, Cantarini L, Liakouli V, Vitale A, Carubbi F, Berardicurti O, Galeazzi M, Valenti $M$ and Giacomelli R: Efficacy of inhibition of IL-1 in patients with rheumatoid arthritis and type 2 diabetes mellitus: Two case reports and review of the literature. J Med Case Rep 9: 123, 2015.

35. Yoshida Y and Tanaka T: Interleukin 6 and rheumatoid arthritis. Biomed Res Int 2014: 698313, 2014.

36. van Schouwenburg PA, Rispens $T$ and Wolbink GJ: Immunogenicity of anti-TNF biologic therapies for rheumatoid arthritis. Nat Rev Rheumatol 9: 164-172, 2013.

37. Miyabe Y, Miyabe C, Iwai Y, Yokoyama W, Sekine C, Sugimoto K, Harigai M, Miyasaka M, Miyasaka N and Nanki T: Activation of fibroblast-like synoviocytes derived from rheumatoid arthritis via lysophosphatidic acid-lysophosphatidic acid receptor 1 cascade. Arthritis Res Ther 16: 461, 2014. 
38. Garcia-Carbonell R, Divakaruni AS, Lodi A, Vicente-Suarez I, Saha A, Cheroutre H, Boss GR, Tiziani S, Murphy AN and Guma M: Critical role of glucose metabolism in rheumatoid arthritis fibroblast-like Synoviocytes. Arthritis Rheumatol 68 : 1614-1626, 2016

39. Calnan DR and Brunet A: The FoxO code. Oncogene 27: 2276-2288, 2008.

40. Qian Z, Ren L, Wu D, Yang X, Zhou Z, Nie Q, Jiang G, Xue S, Weng W, Qiu Y and Lin Y: Overexpression of FoxO3a is associated with glioblastoma progression and predicts poor patient prognosis. Int J Cancer 140: 2792-2804, 2017.

41. Nho RS, Hergert P, Kahm J, Jessurun J and Henke C: Pathological alteration of FoxO3a activity promotes idiopathic pulmonary fibrosis fibroblast proliferation on type i collagen matrix. Am J Pathol 179: 2420-2430, 2011.

42. Czyszek JA, Milkiewicz M, Elias E and Milkiewicz PJG: Proapoptotic protein bim and its upstream activator Foxo3A are overexpressed in primary biliary cirrhosis but not in primary sclerosing cholangitis. Gut 60 (Suppl 1): A227, 2011.

43. Harada Y, Harada Y, Elly C, Ying G, Paik JH, DePinho RA and Liu YC: Transcription factors Foxo3a and Foxo1 couple the E3 ligase Cbl-b to the induction of Foxp3 expression in induced regulatory T cells. J Exp Med 207: 1381-1391, 2010.
44. Lin L, Hron JD and Peng SL: Regulation of NF-kappaB, Th activation, and autoinflammation by the forkhead transcription factor Foxo3a. Immunity 21: 203-213, 2004.

45. Lin H, Dai T, Xiong H, Zhao X, Chen X, Yu C, Li J, Wang X and Song L: Unregulated miR-96 induces cell proliferation in human breast cancer by downregulating transcriptional factor FOXO3a. PLoS One 5: e15797, 2010.

46. Wu Y, Peng H, Cui M, Whitney NP, Huang Y and Zheng JC: CXCL12 increases human neural progenitor cell proliferation through Akt-1/FOXO3a signaling pathway. J Neurochem 109: 1157-1167, 2009.

47. NakaoT,Geddis AE,Fox NEandKaushanskyK:PI3K/Akt/FOXO3a pathway contributes to thrombopoietin-induced proliferation of primary megakaryocytes in vitro and in vivo via modulation of p27(Kip1). Cell Cycle 7: 257-266, 2008.

(†) $\Theta$ This work is licensed under a Creative Common Attribution-NonCommercial-NoDerivatives 4.0 International (CC BY-NC-ND 4.0) License. 